\title{
XIII.
}

\section{Acht Behauptungen Nissl's}

\author{
beantwortet von
}

\section{Dr. P. Kronthal}

in Berlin.

Im Centralblatt für Nervenheilkunde und Psychiatrie vom 15. Maî 1904 hat Nissl einen Artikel gegen meinen Aufsatz "Nervenzelle und Psychose" (Arch. f. Psych. Bd. 38) veröffentlicht. Er stellt 8 beweislose Behauptungen auf, die ich wiedergebe und beantworte. Die Beantwortung machte mir insofern gewisse Schwierigkeiten, als es mir nicht gelang, einen Gedanken, der für die Reihenfolge der Behauptungen bestimmend war, zu finden. Hätte ich einen solchen gefunden, so konnte ich demselben folgen und Antwort logisch an Antwort knüpfen. So aber sah ich mich gezwungen, in der einen Antwort auf die andere zu verweisen; trotzdem liessen sich Wiederholungen nicht gänzlich vermeiden.

Nissl schreibt:

.1. Es ist unrichtig, zu behaupten, dass es im gesunden Centralorgan von Mensch, Hund, Katze und Kaninchen Nervenzellen giebt, die keinen Kern mit wohl differenzirter Kernmembran besitzen, welche den letzteren gegen den Zellleib abgrenzt. Wohl aber existiren artificielle Abweichungen vom Nervenzellenäquivalentbild, wobei die Nervenzellenkerne theils in Auflösung begriffen sind (kïnstliche Schwellung), theils vom Zellleib sich schwer abgrenzen lassen (künstliche Schrumpfung)".

Nervenzellen mit unscharfem Kern sollen in vivo nicht vorkommen, sondern Kunstprodukte sein. Nach der von mir verwendeten Methode werden die wenige $\mathrm{cmm}$ grossen, dem eben getödteten Thiere entnommenen Stücke in ein Gemisch von abs. Alkohol und Eisessig gebracht, in dem sie nach 15 Minuten vollständig fixirt sind. Um starke Diffusionsströme beim Ueberführen in Alc. abs. zu verhüten, wird der Essigsäure-Gehalt langsam herabgesetzt. Man muss die Stücke in Serien von etwa $1,5 \mu$ Dicke schneiden, weil dickere Schnitte so grosse Theilstücke des Kerus geben, dass ein Urtheil über seine allsei- 
tigen Grenzen nicht angängig ist. An derartig gewonnenen Serien kann man sehr scharf beobachten, wie in vielen Nervenzellen ein Kern theilweise oder auch allseitig nicht scharf abgrenzbar, öfter überhauft nicht rorhanden ist.

Der Nachweis derartiger Zellen gelingt durchaus nicht nur mit der hier angegebenen Methode, sondern nach Verwendung der verschiedensten, ganz different wirkenden Reagentien. Bei allen zur Fixation des Nervensystems verwendeten Methoden finden sich in zahlreichen Nervenzellen die Grenzen zwischen Kern und Protoplasma in geringerem und grösserem Umfange unscharf bis fehlend.

Aus dem mikroskopischen Bilde des fixirten Gewebes ziehen wir Schlüsse auf den Zustand des lebenden Gewebes. Wir sprechen keiner Fixationsmethodè absoluten Werth zu, sondern controliren die Bilder, welche eine Methode giebt, durch die mit anderen Methoden erbaltenen. Wenn von gleichartigen, nach den verschiedensten Methoden fixirten Zellen ein Theil stets unscharfe bis fehlende Kernconturen zeigt, haben wir zu schliessen, dass in vivo diese Zellart zum Theil mangelhafte Kerngrenzen hat. Dieser Schluss ist geboten und wird analog auf dem gesammten Gebiete der Histologie stets gemacht. Wer im Gegensatz dazu die Behauptung aufstellt, dass nach den verschiedensten Methoden zu beobachtende, gleichartige Bilder Kunstproducte seien, verkennt die Grundsätze, wach denen alle histologischen Bilder gedeutet werden. Er deutet nicht histologische Bilder, sondern er speculirt: Weil eine grosse Anzahl Zellkerne dieser Zellenart scharfe Grenzen hat, sind die unscharfen Grenzen in anderen, gleichartigen Zellen Kunstproduct. Dieser Schluss ist unberechtigt, weil derartige Zellen mit unscharfen Kerngrenzen nicht gelegentlich bei einer, sondern regelmässig bei allen Methoden zu sehen sind.

,2. Es ist ein mir unfassbarer Irrthum, wenn man behauptet, dass die centralen Nervenzellen sterbende resp. gestorbene Organismen sind und keine Lebensänsserungen haben. Durch die einfachsten, nur geeignet angeordneten Experimente kann man sich überzeugen, dass die Nervenzellen auf äussere Einflüsse reagiren, Substanzen verlieren, ihre Formen verändern und sich wieder vollständig zurückbilden können".

Die centralen Nervenzellen sind keine Organismen, weil sie

a) keine Nahrungsstoffe aufuehmen und verarbeiten. Beweis efr. meine Arbeit "Von der Nervenzelle etc." S. 68 u. folg., mein Vortrag "Biologie und Leistung der centr. Nervenzelle", Neurol. Centralbl. 1903, No. 4. Sep-Abdr. S. 2. Ein Körper, der keine Nahrung verarbeitet, ist kein Organismus. Die Annahme, es gäbe gewisse Stoffe, die speciell 
von der Nervenzelle aufgenommen und verarbeitet würden, wie die Nar-cotica, ist falsch. Beweis: a) Lebewesen ohne Nervenzellen reagiren auf Narcotica genau so wie Lebewesen mit Nervenzellen. $\beta$ ) Isolirt man einen Theil eines Lebewesens vom centralen Nervensystem, soreagirt dieser Theil auf Narcotica wie früher im Zusammenhang mit dem Nervensystem. Von der Nervenzelle geht die Wirkung der Narcotica also nicht aus.

b) Weil die Yervenzelle sich niemals theilt, weder beim Embryo ${ }_{r}$ noch jemals später (cf. "Nerrenzelle und Psychose", Sep.-Abdr. S. 6). Ein Körper, der sich niemals fortpflanzt, ist kein Organismus.

c) Weil die Eigenschaften der Lebewesen; die man als Leistung der Nervenzelle ansieht, die Psyche, sich auch bei Lebewesen findet, die keine Nervenzellen haben. Psyche kann also nicht Leistung der Nervenzelle sein. Eine andere Leistung ist den Nervenzellen nie zugesprochen worden. Sie leisten also, als Organismen betrachtet, nichts. Ein Organismus, der nichts leistet, ist ein Unding.

d) Wäre die Nervenzelle ein Organismus, so müsste nothwendigerweise nach Verletzung oder Erkrankung desjenigen Organs, welches die meisten Nervenzellen birgt, eine Verminderung an Leistungen der Nervenzellen, der Psyche, zu constatiren sein. Es kommen ziemlich umfangreiche Verletzungen und Erkrankungen des Gehirns ohne psychische Störungen vor. Psyche kann also nicht Leistung der Nerrenzelle sein. Eine andere Leistung ist ihr nie zugesprochen worden. Ein Organismus, der nichts leistet, ist ein Unding.

Noch niemals hat Nissl oder sonst ein Mensch gesehen, dass Nervenzellen "Substanzen verlieren, ihre Formen verändern und sich wieder: vollständig zurückbilden" aus dem einfachen Grunde, weil noch niemals ein Hensch eine centrale Nervenzelle in ihrer Thätigkeit beobachtet hat. Die Beobachtungen, die Nissl nebst vielen Anderen gemacht hat und die zu leugnen mir nicht eingefallen ist (cf. Nervenzelle and Psychose, Arch. f. Psych., Bd. 38, Sep.-Abd. S. 27, S. 49 etc.) lauten: Nach Entfernung peripherer Theile, nach gewissen Vergiftungen, lang anhaltenden Temperaturänderungen finden sich Nervenzellen, die Abweichungen von den Nervenzellen zeigen, die sich normaler Weise finden. Dies erklärt sich sehr einfach. Sind Fasern degenerirt, fehlen Fasern, so muss sich die Form der Nervenzellen ändern, weil diese entstehen, indem Leukocyten die Fasern umfliessen; sind die Leukocyten, die Transporteure von Fremdstoffen, mit Giften beladen, so werden die Nervenzellen als Product, Untergangsform der Leukocyten, Abweicbungen zeigen. Das glaube ich ausfïhrlich genug beschrieben zu haben, um es 
fassbar zu machen. Weshalb die Nervenzellen aus Leukocyten entstehen und entsteben müssen, s. Antwort zu 5.

$n^{3}$. Es widerspricht einer leicht zu constatirenden Thatsache, wenn man behauptet, dass mit Farbbasen sich tingirende Substanzen nur im Zellkern, nicht aber auch im Zellleib verschiedener Zellarten nicht nervöser Natur auftreten. "

Die Nervenzelle ist zu allen anderen Zellen, die sich bei hochstehenden Vertebraten normaler Weise findet, gegensätzlich, weil ihr Protoplasma regelmässig chromatische Massen enthält. Ich sehe mit höchstem Interesse der Mittheilung Nissl's entgegen, welche andere physiologisch vorkommende Zellart chromatine Substanzen in ihrem Protoplasma gesetzmässig aufweist. Wohl kenne ich Drüsenzellen mit basophilen Secreten. So lange die Zelle ihr Secret noch nicht ausgestossen hat, färbt es sich selbstredend auch in der Zelle mit Farbbasen. Die leere Drüsenzelle nimmt Farbbasen nur im Kerne an.

Für jede Zelle giebt es einen Moment, in dem sie in ibrem Protoplasma Kernstoffe enthält, der Moment nämlich, wenn sie gestorben, in Auflösung begriffen ist. Dann kommt es zu Zersetzungen. Die Sonderung zwischen Kern und Protoplasma hört auf. Die Menge Kernsubstanz, die dann im Protoplasma auftritt, kann aber nie mehr betragen als der Kern enthielt. Und dies ist das zweite ungemein Charakteristische für die Nervenzelle: In ihrem Protoplasma finden sich chromatine Substanzen, die an Quantität die chromatinen Massen des Kernes sehr oft um vieles übertreffen.

„4. Es ist eine durch und durch irrth ümliche Behauptung, dass die mit basischen Farben sich tingirenden Substanzportionen des Nervenzellleibes bei der Auflösung von Kernen entstehen, resp. sich aus dem Kernchromatin herleiten."

Nach meiner Auffassung vom Entstehen der Nervenzellen (s. Antwort auf 5) ergiebt sich die Herkunft der chromatischen llassen im Protoplasma aus fremden Kernen als nothwendige Consequenz. Eine andere annehmbare Erklärung für die Herkunft dieses Chromatins kenne ich nicht. Dabei bleibe nicht unbemerkt, dass "Chromatin" kein chemischer Begriff ist und dass die basischen Farbstoffe durchaus keine specifischen Reagentien für die in den Kernen vorkommenden Eiweissarten sind.

Es giebt Thiere, deren Nervenzelle sehr wenig bis gar kein Chromatin im Protoplasma birgt. Diese Nervenzellen können nicht Verschmelzungsproducte von anderen Zellen sein. Es können nicht alle Jervenzellen Verschmelzungsproducte von Lenkocyten sein aus dem sehr einfachen Grunde, weil es Thiere zwar ohne Leukocyten, ohne Kreis- 
lauf, wohl aber mit Nervenzellen giebt. Das Nervensystem ist eine Reiz leitende Verbindungsconstruction. Diese Construction wird bei den verschiedenen Arten sehr verschieden sein. Je besser sie ist, desto höher steht geistig die Art.

„5, Es ist mir unbegreiflich, wie jemand behaupten kann, dass im gesunden Centralorgan Leukocyten die Capillaren verlassen und das Gewete durchwandern. Eine solche Angabe kann nur jemand machen, der sich mit der Glia nicht näher beschäftigt hat."

Seit den Arbeiten von Cohnheim, v. Recklinghausen, Arnold, Stricker u. A. steht unbestritten fest: Leukocyten durchsetzen die Capillarwände, durchwandern Gewebe. Das Gehirn, speciell die graue Substanz, ist besonders reich an besonders feinen Gefässen. Das Gehirn ist eine weiche Masse. Die Annahme, auch in Gehirn verlassen Leukocyten die Capillaren und wandern in das Gewebe ein, entspricht einer grundsätzlichen Forderung der Logik. Würde man im centralen Nervengewebe keine Leukocyten finden, so wäre dies ein unerklärbares Vorkommen.

Den inductiven Beweis für das Auswandern der Leukocyten in das Gehirn kann ich nicht führen, weil ich zur Zeit keine Möglichkeit sehe, nur mikroskopisch wahrnehmbare Vorgänge in einem unverletzten Gehirn sichtbar zu machen. Deductive Beweise für das Auswandern von Leukocyten ins centrale Nervensystem:

a) Es giebt im centralen Nervensystem zahlreiche Zellen, dis morphologisch und nach ihrem mikrochemischen Verhalten nit den Lenkocyten übereinstimmen. Man findet diese Zellen sowohl dicht neben den Gefässen als im Gewebe.

b) Es sind im centralen Nervensystem viele derartige Zellen an den Orten, wo viele Capillaren sind.

c) Farbkörper (Methylenblau, Nentralroth), die in den Kreislauf gelangen, finden sich in Zellen des centralen Nervensystems. Den Transport von Farbstoffen besorgen, soweit sie sie aufnehmen, die Leukocyten (s. Antwort auf 7).

d) Die Substanz an den Orten mit den vielen Capillaren, die graue Substanz, besteht ans marklosen Fibrillen. Diese Fibrillen, zu ungemein feinem Gewebe rerflochten, müssen die Lenkocyten in ihrer Wanderung behindern. Die Leukocyten umfliessen die Fibrillen, wie sie erfahrungsgemäss stets mit Fremdkörpern thun. Deshalb finden wir die Zellen von Fibrillen durchsetzt. Die Lenkocyten, die von Nervenfibrillen festgebalten werden, sind Nervenzellen geworden, die von der Glia festgehaltenen Gliazellen. Deshalb finden wir die Gliazellen von Gliafasern durchzogen. Diese Fasern sind ungleichmässiger, 
gröber als die Nervenfibrillen. Sehr charakteristischer Weise zeigen sich bäufig Gliazellen, die nicht von den Fasern durchzogen werden, sondern ihnen nur anliegen und zwar oft so, dass die Zelle in einem von Fasern gebildeten Winkel liegt. Dieses Verhalten hat mir schon lange die Annahme, Gliazelle und Gliafaser seien eine organische Einheit, verdächtig erscheinen lassen. Der wandernde Leukocyt wird von Faserwinkeln natürlich leicht anfgehalten. Gliazelle und Gliafaser sind genau so wenig eine organische Einheit als Nervenzelle und Nervenfaser.

Es ist mir heute unbegreiflich, wie ich mich von den negativen Bildern der Golgi'schen Methode einst konnte verleiten lassen, entgegen einer Denknothwendigkeit, entgegen den deutlichen Bildern als zuverlässig erkannter lletboden, den kurzsichtigen Satz zu glauben: Im Centralnervensystem giebt's keine Leukocyten. Wenn es nur je den Schein eines Beweises für diesen Satz gegeben hätte! Auch nicht der kleinste Grund ist bekannt, der Leukocyten hindern sollte, die Hirncapillaren zu verlassen, in das weiche Hirngewebe einzuwandern. Niemals hat jemand bewiesen, dass das Urtheil über unseren Sinneseindruck, nach dem die kleinen Zellen im Centralnervensystern mit den Leukocyten übereinstimmen, falsch ist. An diese Uebereinstimmung glaubten die besten Histologen - bis jene unglückselige Golgi'sche Methode kam. Der alte Glaube war richtig, die neue Methode falsch. Was wir bei der Golgi'schen Methode gefärbt sehen, sind keine körperlichen Gebilde sondern Ausgüsse von Hohlräumen. Das wird wohl kaum noch bezweifelt.

Giebt man zu, dass Leukocyten die Hirncapillaren verlassen und in das Gewebe einwandern, so entwickeln sich die Consequenzen mit mathematischer Genauigkeit. Wir sehen Fäden in den Zellen, weil die Zellen vom Fasergewirr festgehalten werden. Die für ein freies Leben organisirte, einheitlich geschlossene Zelle ist jetzt fixirt, ibr Körper von ihr fremden Körpern, den Fäden durchsetzt. Dies muss eine tiefe Schädigung ihrer Lebensbedingungen sein. Sie stirbt. Deshalb sehen wir die scharfen Grenzen zwischen Kern und Protoplasma schwinden. Als todter, zähflüssiger Körper folgt die Nervenzelle für solche Körper geltenden Gesetzen. Sie confluirt mit ihr gleichen Körpern. Deshalb sehen wir die Zellen verschmelzen. Als todter Körper wird sie resorbirt. Deshalb bleibt trotz dauernden Auswanderns der Leukocyten die Zahl der Nervenzellen im Ganzen stets dieselbe. Als todter Körper kaun sie keine Nahrungsstoffe umsetzen, als Organismus nichts leisten. Die Nervenzelle setzt keine Nahrungsstoffe um. Sie leistet als Organismus nichts. Alle ihr zugeschriebenen Leistungen des Metazoon erklären sich um vieles naturwissenschaftlicher, einfacher, logischer, wider- 
spruchsfreier, wenn man, wie es sich als nothwendig erweist, absieht, die Nervenzelle als Organismus aufzufassen.

,6. Es ist für mich unbegreiflich, dass jemand, der sich unter Benutzung der modernen Hülfsmittel mit den Nervenzellen eingehend beschäftigt hat, erklären kann, dass die Nervenzellen das Product verschmolzener Leukocyten sind, fortwährend untergehen und dauernd durch Verschmelzung von Leukoayten neu entstehen. "6

Eine Antwort erübrigt sich durch die vorangegangenen Antworten.

"7. Es ist unrichtig, wenn behauptet wird, dass im Centralorgan der Transport von Farbstoffkörnchen allein durch Leukocyten besorgt wird und dass dadurch die Erscheinung der Ehrlich'schen vitalen MethylenblauFärbung sofort verständlich wird."

In meinem Aufsatz "Nervenzelle und Psychose" (Sep.Abd. S. 10) schreibe ich: "Den Transport von Farbstoff körpem im Organismus besorgen, wie bekannt ist, die Lenkocyten." In meinem Vortrag "Die Beziehungen des Nervensystems zur Psyche" (Neurol. Centr.-Bl. 1904, No. 4) sage ich: "Den Transport von Farbkörpern im Blute besorgen, wenn sie die Farbstoffe aufnehmen, die Leukocyten." Weder spreche ich davon, dass die Leukocyten "allein" den Transport von Farbstoffen besorgen, noch schränke ich meine Aussage auf das Centralorgan ein.

Entstehen, wie ich annehme, die Nervenzellen aus Leukocyteu, so ist es mir sofort verständlich, weshalb Farbstoffkörper, welche in die Blutbahn gelangen, sich in den Nervenzellen wiederfiuden. Dass auch die peripheren Nerven und Nervenendigungen sich nach NethylenblauInfusionen färben, ist natürlich kein Beweis gegen meine Theorie. Einmal bestehen, wie ich in meinen Arbeiten mehrfach betont habe, sehr innige Stoffwechselbeziehungen zwischen Nervenzelle und peripherem Nerv, sodass Stoffe aus jener in diesen übertreten, andererseits entbält wohl der Nerv Stoffe, die Methylenblau binden, wie es ihm auch zugeführt wird. Nan findet aber nicht nur Methylenblau, sondern auch andere Farbstoffe, überhaupt Körper, die man in den Leukocyten findet, in der Nervenzelle wieder. Dies beweisen namentlich nenere Arbeiten der Bakteriologen.

"8. Es ist ein Irrthum zu sagen, dass keine wesentlichen Differenzen zwischen den Fortsätzen einer Nervenzelle bestehen. Abgesehen von den morphologischen Differenzen zwischen dem Axencylinderfortsatz und den Protoplasmafortätzen zeigt sich darin ein sehr wesentlicher Unterschied, dass die Neurofibrillen anscheinend unverändert den Neuriten verlassen und zuFibrillen eines Axencylinders werden, während es noch niemandem gelungen ist, eine Neurofibrille über das Gebiet der Dendriten hinaus zu verfolgen."

Dass morphologische Differenzen zwischen dem Neurit und dem Lendriten bestehen, beschreibe ich: „In einem Fortsatz liegen die 
Primitivfibrillen enger aneinander." (Nervenzelle und Psychose. Sep.Abd. S. 28.)

Das Geschick der Fibrillen, soweit sie über die Zellfortsätze hinausgehen, also jenseits des Zellprotoplasmas, habe ich in meinen sämmtlichen Arbeiten nie berücksichtigt. Was ans den Fibrillen wird, wenn sie den Neuriten resp. den Dendriten verlassen, erörtere ich nirgends. Im Gegentheil sage ich (Nervenzelle und Psychose, Sep-Abd. S. 29) ausdrücklich von den verschiedenen Fortsätzen: „Da die einen Fibrillen wie die andern nicht organische Theile der Zelle vorstellen, sind sie rïeksichtlich ihrer Beziehungen zur Zelle gleichwerthig." Ich bezeichne sie als gleichwerthig "rücksichtlich ihrer Beziehungen zur Zelle" and schreibe weiter ausdrücklich, dass die Einen zur Peripherie gelangen, die andern im centralen Nervensystem zur Auflösung kommen, ohne in die Erörterung einzutreten, welche zur Peripherie gelangen.

Bei dieser Gelegenheit möchte ich bemerken, dass die Annahme, jede Nervenzelle habe einen Neuriten, sicher irrig ist. Die Behauptung, jede Nervenzelle habe einen Neuriten, gründet sich nicht etwa auf Beobachtung. Gerade die Beobachtung lehrt, dass an sehr vielen Zellen ein Nenrit nicht nachzuweisen ist. Man kann sich davon an Serienschnitten und an isolirten Zellen sehr gut überzengen. Dass ein Neurit an vielen Zellen nicht zu finden ist, erklärte man aber nicht durch sein Fehlen, sondern durch die Entschuldigung, die betreffende Zelle sei durch den Schnitt unglüeklich getroffen, der Neurit sei abgebrochen etc. Das häufige Fehlen des Neuriten erklärt sich nach meiner Auffassung rom Entstehen der Nervenzelle einfach. Im centralen Nervensystem sind, wie es die Fibrillenpräparate lehren, einzelne Fibrillenbündel enger aneinander gepackt als die Mehrzahl. Umfliesst ein Leukocyt resp. ein Leukocytenconglomerat neben in gewöhnlicher Art gelagerten Fibrillenbündeln auch ein solch enger gepacktes, so hat die Nervenzelle einen Neurit, anderenfalls nicht.

Man darf bei der Darstellung des Nervensystems uie von der Nervenzelle ausgehen. Sie ist das Secundäre. Ontogenetisch wie phylogenetisch tritt zuerst die Nervenfaser auf.

Es sind fünf Thatsachen an den Zellen des Centralnervensystems zu constatiren: 1. Zahlreiche kleine Zellen gleichen morphologisch und mikrochemisch den weissen Blutkörpern. 2. Alle Zellen werden von zahlreichen Fibrillen durchzogen, die in ihnen nicht enden und nicht beginnen. 3. Grössere Zellen ungreifen mit ihrem Protoplasma kleinere Zellen theilweise bis vollständig. 4. In dem Protoplasma der grossen Zellen finden sich regelmässig chromatine Substanzen, d. h. Substanzen, die sich regelmässig sonst nur in den Kernen von Zellen finden. 
5. Kern und Protoplasma sind vielfach nicht scharf getrennt, sind oft nicht gesondert.

Wer sich von Thatsache 1 überzeugen will, braucht in gefärbten Präparaten nur sorgfältig die Leukocyten und die kleinen Zellen zu vergleichen. Thatsache 2 wird wohl jetzt kein Mensch mehr bezweifeln, der Präparate nach der neuen Silber-Methode Ramon y Cajal's gesehen hat. Thatsache 3 ist längst bekannt. Man findet die kleineren Zellen als "Trabantenzellen" erwähnt oder die grossen als "mehrkernig" beschrieben. Diese Mehrkernigkeit wird mitunter als normales Vorkommen, mitunter als Degenerationszeichen aufgefasst. Von Thatsache 4 kann sich seit der Entdeckung Flemming's Jeder durch einfache Kernfärbung überzengen. Wer Thatsache 5 erkennen will, fixire nach den verschiedensten Methoden und schneide Serien von zureichender Dünne.

Wollen wir uns eiu Bild von dem Wesen der Nerrenzelle und damit des centralen Nervensystems machen, so dürfen wir keine dieser Thatsachen unberücksichtigt lassen. Der A páthy zuerst unzweifelhaft gelungene Nachweis, dass die Fibrillen in der Nervenzelle nicht Anfang und nicht Ende haben, war für die gesammte Anatomie, Physiologie, Pathologie, pathologische Anatomie des Nervensystems im höchsten Grade unbequem. Denn auf die Annahme, der periphere Nerv sei Fortsatz der Nervenzelle gründeten sich alle Vorstellungen von der Leistung der Nervenzelle. Nun lehren die modernen Fibrillenpräparate unzweifelhaft, dass die Fibrillen, die Haupttheile der peripheren Nerven, nicht Fortsătze der Zelle sind. Wie Allen, die sich mit der Nervenzelle beschäftigen, erging es auch mir. Ich konnte das Durcheilen der Fibrillen durch die Nervenzelle and meine bisherigen Vorstellungen von den Beziehungen der peripheren Nerven zur Nervenzelle in eine einheitliche Auffassung nicht zusammenbringen. Die lückenlosen Bilder von ausgezeichneter Schürfe, welche die Alkohol-Eisessig. Methode giebt, liessen mich die Nervenzelle als ans Leukocyten entstanden sehen. Mit dieser Erkenntniss war sofort der Boden für eine Auffassung gewonnen, die alle von der Nervenzelle bekannten Thatsachen nach Ursache und Wirkung logisch ordnen liess. So kam ich zu dem folgenschweren Schluss: Die Nervenzelle ist kein Organismus. Die Richtigkeit desselben musste geprüft werden. Wo auch immer man die Prüfung versuchte, es zeigte sich, dass diese Auffassung von der Nervenzelle unseren Sinneswahrmungen gerecht wird. Man kann den metaphysischen Begriff der Psyche, der in den Naturwissenschaften einen Platz nicht haben sollte, entbehren und statt seiner einen physiologischen Begriff, die Summe der Reflexe, einführen. 
Wir haben als Naturforscher die Aufgabe unsere Sinneswahrnehmungen nach dem supponirten Gesetz von der Causalität zu deuten. Sind mehrere Deutungen vorhanden, so entscheiden wir uns für diejenige, die unsere Wahrnehmungen an der Hand der Naturgesetze einfacher, logischer, widerspruchsfreier erklärt, weil sie wahrscheinlicher das Wesen der Dinge trifft. Die alte Hypothese, nach der die Nervenzelle ein Organismus und ihre Leistung die Psyche ist, kann ich als richtig nicht mehr anerkennen. Wie sie entstanden ist und entstehen musste, glaube ich in meinen Arbeiten gezeigt zu haben. Ich gebe diese Hypothese auf, weil eine bessere möglich ist.

Auf die grossen Worte, mit denen Nissl seine Behauptungen in dem ihm eigenen Stile einleitet und schliesst, gehe ich nicht ein, weil ich eine Form der Antwort nicht finden kann, die Zweifel an ihrer Sachlichkeit ausschliesst. 